Journal Homepage: $-\underline{w w}$ w.journalijar.com
NTIERNATIONAL JOURNAL OF
ADVANCED RESEARCH (IJAR)
ISSN NO. 2320-5407
DOI URL: http://dx.doi.org/10.21474/IJAR01/2722

RESEARCH ARTICLE

\title{
LOCALISATION INHABITUELLE DE LA TUBERCULOSE OSTEOARTICULAIRE (TALUS) : A PROPOS D'UN CAS.
}

\author{
O. Azriouil, M. Daoudi, J. Boukhris, O. Margad and K.I Koulali. \\ Service de Traumatologie-Orthopédie, Hôpital Militaire Avicenne, Marrakech, Maroc.
}

\section{Manuscript Info}

.........................

Manuscript History

Received: 15 November 2016

Final Accepted: 17 December 2016

Published: January 2017

Key words:-

Bone tuberculosis, talus.

\section{Abstract}

The talar tuberculosis is a rare bone localization of Koch bacillus. We report the case of a 50-year-old woman, with no special medical history, who complained of pain in the right foot with chronic evolution. Pathology study of a Bone biopsy led to the diagnosis of talar tuberculosis. A 6-months anti-bacillary treatment was given. The risk of degeneration compromises the functional future of the ankle joint.

Copy Right, IJAR, 2016,. All rights reserved.

\section{Introduction:-}

La tuberculose ostéo-articulaire représente environ 35\% des atteintes extra pulmonaires et environ 1 à $3 \%$ de tous les cas de tuberculoses $[1,2]$. La localisation à la cheville et au pied constitue $2 \%$ des formes squelettiques $[3,4]$. La forme talienne est une entité très rare dont le pronostic dépend de la précocité du diagnostic et l'instauration du traitement spécifique.

Nous rapportons l'observation d'une tuberculose ostéoarticulaire de localisation talienne avec revue de la littérature.

\section{Observation: -}

Il s'agissait de Madame M.R âgée de 50 ans, femme au foyer sans antécédents pathologiques particuliers. Elle s'est présentée dans notre formation pour douleur, tuméfaction et impotence fonctionnelle du pied droit avec une fistule purulente à la face externe de la cheville.

Le début de la symptomatologie remontait à quatre mois, avec l'apparition d'un gonflement douloureux de la cheville qui a été considéré comme une entorse bénigne négligée et traité comme telle. Deux mois plus tard, était apparue une fistule latérale avec issue d'un liquide d'aspect purulent, le tout évoluait dans un contexte d'apyrexie et de conservation de l'état général.

Devant ce tableau, la patiente avait consulté une autre formation hospitalière où elle avait bénéficié d'un bilan biologique standard et d'un examen bactériologique du liquide purulent. Cependant, les résultats n'étaient pas concluants, avec absence d'hyperleucocytose, une protéine-C-réactive négative et un examen direct négatif du prélèvement.

La patiente a été mise sous antibiothérapie à base de pénicilline $\mathrm{M}$, mais sans amélioration notable.

A son admission dans notre service, l'examen clinique retrouvait (figure 1), une tuméfaction et une fistule active à la face externe de la cheville qui donnait du liquide séreux et une boiterie à la marche, l'articulation tibio-astragalienne 
était libre. Les radiographies standards de face et de profil de la cheville (figure 2), montraient une lésion lacunaire de la partie supéro-médiale du corps du talus, grossièrement arrondie avec ostéosclérose périphérique. La tomodensitométrie de la cheville avait confirmé l'atteinte ostéo-articulaire et avait objectivé la présence d'une lésion ostéolytique du talus (figure 3).

La biopsie osseuse fut alors réalisée. L'abord a été latéral avec excision des tissus inflammatoires et nécrotiques, résection du trajet fistuleux et curetage biopsique talien large. Un nétoyage articulaire au sérum physiologique a été pratiqué et une immobilisation par attelle postérieure a été mise en place.

L'histologie permit d'affirmer le diagnostic de tuberculose par la présence de nombreux granulomes épithélioides et giganto-céllulaire centrés d'une nécrose caséeuse, tandis que l'analyse bactériologique était non contributive avec mise en évidence d'une flore bactérienne polymorphe où prédominait le Pantoea Agglomerrans. Un bilan d'extension n'a pas montré de localisation pulmonaire ou extra pulmonaire.

Après un bilan hépatique, rénal et hématologique, un traitement antibacillaire a été instauré pendant six mois à base de Rifampicine (R) : $5 \mathrm{mg} / \mathrm{kg} / \mathrm{j}$, d'Isoniaside (H) : $10 \mathrm{mg} / \mathrm{kg} / \mathrm{j}$, de Pirazinamide (P) : $25 \mathrm{mg} / \mathrm{kg} / \mathrm{j}$ et d'Ethambutol (E) : $20 \mathrm{mg} / \mathrm{kg} / \mathrm{j}$, avec deux mois de RHZE et quatre mois de RH.

La fistule s'est tarie en quatre semaines, mais la patiente garde toujours des douleurs séquellaires à la marche au dernier contrôle.

\section{Iconographies:-}

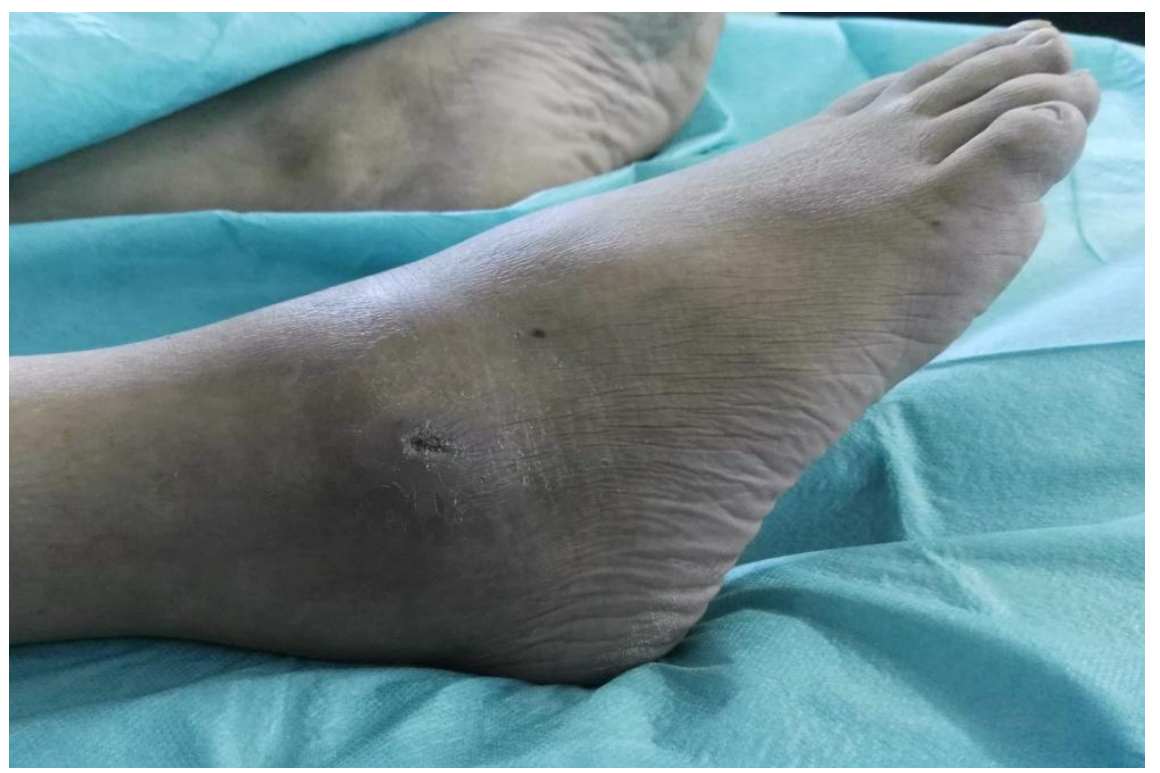

Figure 1:- Tuméfaction avec fistule à la face externe de la cheville droite. 


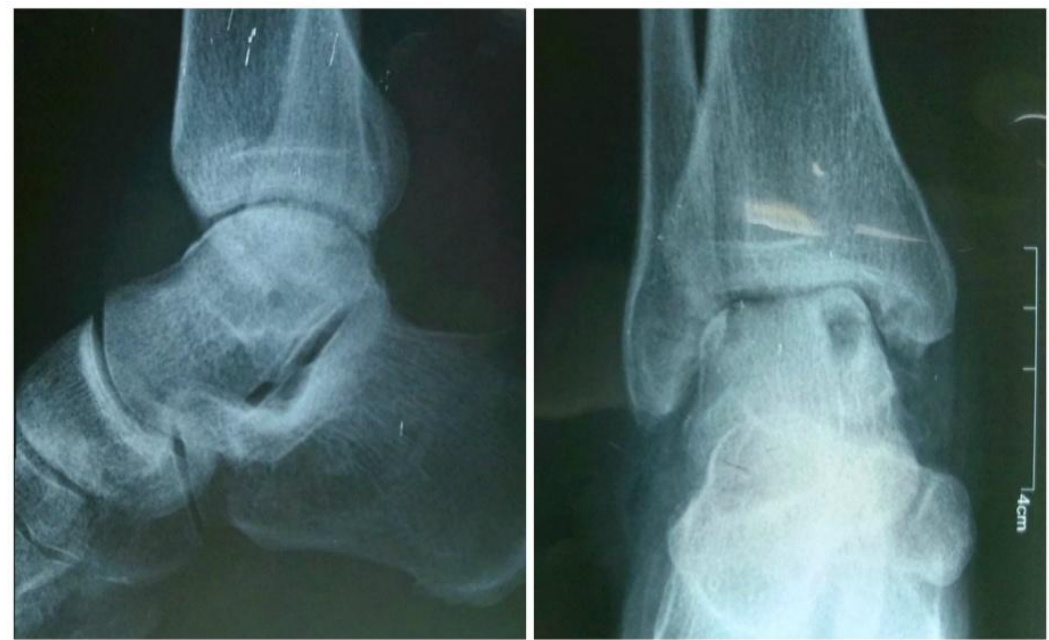

Figure 2:- Radiographie standard de face et de profil ; lésion lacunaire du talus.

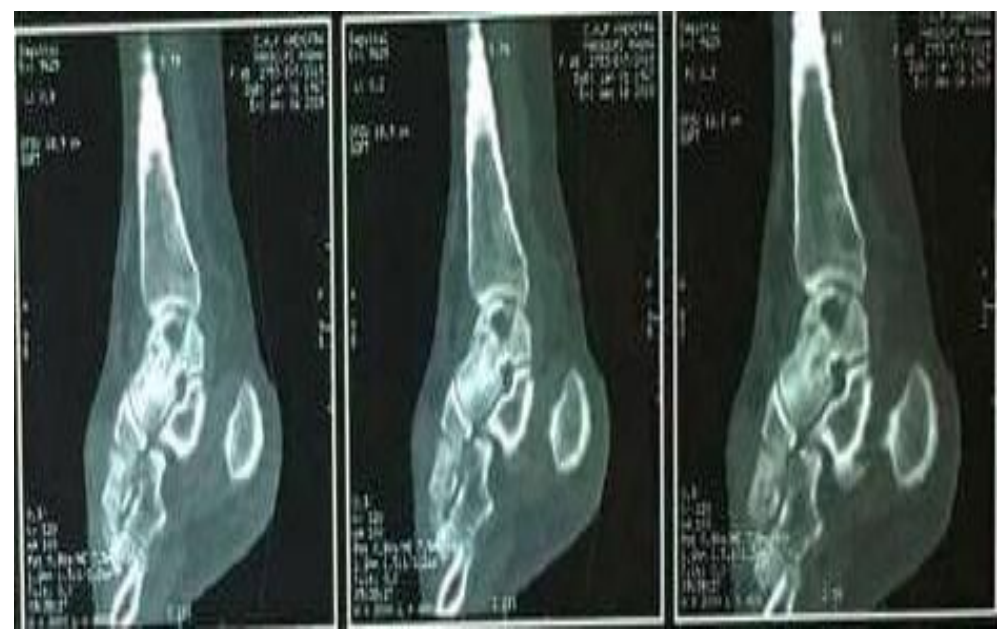

Figure 3:- La tomodensitométrie en coupes sagittales ; lésion ostéolytique du talus.

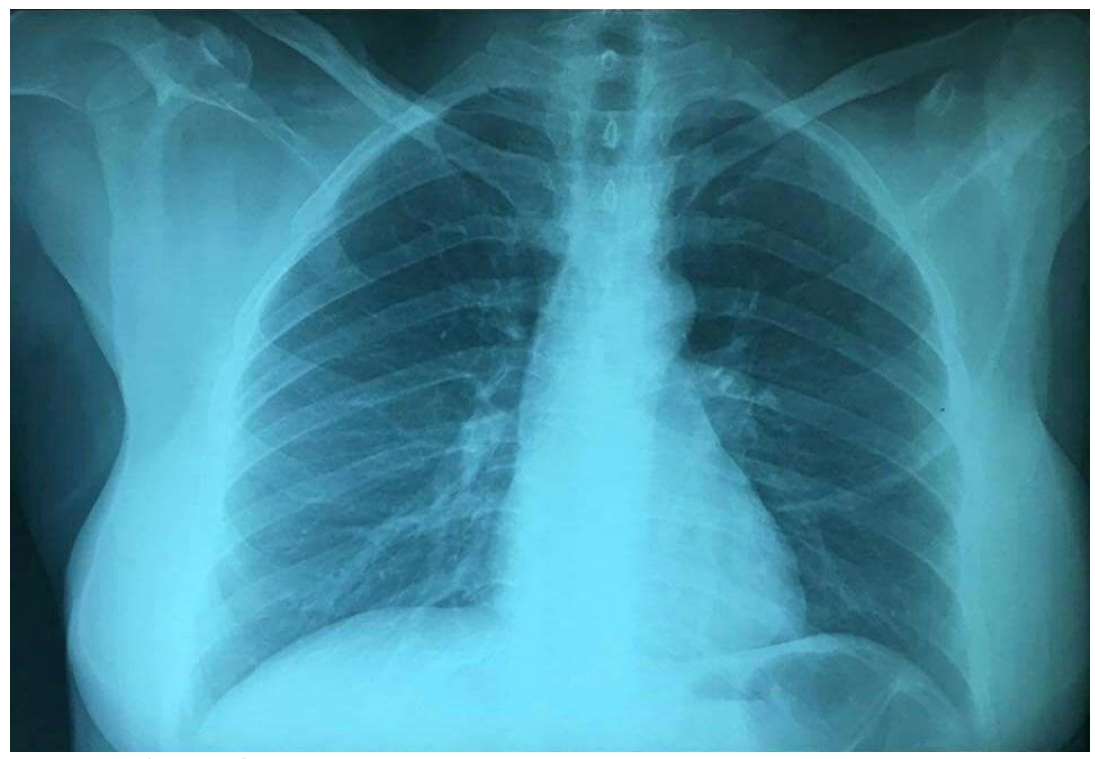

Figure 4:- Radiographie du thorax de face normale. 


\section{Discussion:-}

Selon les données de l'Organisation Mondiale de la Santé en 2014, 9,6 millions de personnes ont développé la tuberculose. Au Maroc, un total de 30.636 cas de tuberculose, toutes formes confondues, a été notifié en 2015. La proportion de la forme extra-pulmonaire était la plus élevée en 2015 , avec $52 \%$ des cas, contre $48 \%$ des cas de tuberculose pulmonaire [6].

Les formes extra-pulmonaires sont dominées par l'atteinte rachidienne qui représente plus de la moitié des cas [7]. La localisation à la cheville est très rare et ne représente que $2 \%$ des atteintes squelettiques. Zacharia et al. [5] ont revu une série de 14 cas de tuberculoses de la cheville, la localisation talienne et calcanéenne était la plus fréquente. Chez notre patiente l'atteinte était talienne.

La rareté de cette affection et les manifestations cliniques aspécifiques expliquent un retard diagnostique pouvant aller de quelques semaines à plusieurs mois [1]. Les principales manifestations cliniques sont la douleur, le gonflement et l'impotence fonctionnelle. Les signes d'imprégnation tuberculeuse sont rares. A des stades tardifs, on peut observer une fistulisation sur la peau [5].

La radiographie osseuse standard est aspécifique. Au début, la tuméfaction des tissus mous et l'ostéopénie peuvent être présentes avant les signes tardifs de destruction osseuse. La radiographie du thorax n'est pas d'une grande spécificité car plus de $80 \%$ des patients n'ont pas de tuberculose active concomitante [8]. Dans notre étude la radiographie du thorax était normale (figure 4). La tomodensitométrie est en retard par rapport à l'imagerie par résonnance magnétique pour la détection de l'atteinte osseuse et bien moins performante pour l'atteinte de tissus mous [8].

La biopsie est le seul élément clé du diagnostic, suivie d'une analyse histologique et bactériologique. La culture du prélèvement biopsique n'est pas toujours concluante et peut nécessiter plusieurs semaines à se positiver [9]; l'aspiration du liquide articulaire en cas d'atteinte associée de l'articulation parait insuffisante pour étayer le diagnostic [10]. Les produits de drainage des fistules sont généralement contaminés par d'autres micro-organismes [10]. Cela a été le cas chez notre patiente où seule l'étude anatomo-pathologique du produit de curetage talien a permis de poser le diagnostic formel de tuberculose du talus.

Le traitement est essentiellement médical par les antibacillaires, il permet de limiter l'évolution vers les séquelles douloureuses et les déformations, la majorité des lésions guérissent en 6-12 semaines sous le traitement médical. Le traitement chirurgical, quant à lui, ne peut en aucun cas remplacer le traitement médical. Il trouve sa place, premièrement, dans l'obtention des prélèvements anatomopathologiques. Deuxièmement, il est indiqué en cas d'insuffisance du traitement médical, lorsque persiste une synovite, une fistule ou un abcès. La chirurgie interviendra enfin dans le cadre des corrections de déformation séquellaire importante et douloureuse [11,12].

\section{Conclusion:-}

La tuberculose ostéoarticulaire, isolée, de la cheville est une entité rarement rapportée dans la littérature, l'atteinte du talus est exceptionnelle. C'est une localisation grave et de diagnostic difficile, pouvant mettre en jeu le pronostic fonctionnel du pied.

\section{Conflits d'intéret:-}

Les auteurs déclarent ne pas avoir de conflits avec ce manuscrit.

\section{Contributions des auteurs:-}

Tous les auteurs ont lu et approuvé la version finale du manuscrit. 


\section{References:-}

1. Yombi JC, Vandercam B, Cornu O, Lecouvet F, Leemrijse T. Ostéoarthrite tarsienne: une localisation rare de la tuberculose. Rev Chir Orthop Reparatrice Appar Mot. 2007 Nov;93(7):740-5.

2. Dhillon MS, Nagi ON. Tuberculosis of the foot and ankle. Clin Orthop. 2002;398:107-113.

3. Vohra R, Kang HS, Dogra S, Saggar RR, Sharmua R : Tuberculous osteomyelitis. J Bone Surg (BR), 1997, 79, 562-566.

4. Watts HG, Lifeso RM: Current concepts review. Tuberculosis of bones and joints. J Bone Surg (Am), 1996, 78, 288-298.

5. Zacharia TT, Shah JR, Patkar D, Kale H, Sindhwani V. MRI in ankle tuberculosis: Review of 14 cases.Australas Radiol. 2003 Mar;47(1):11-6.

6. Situation épidémiologique de la tuberculose au Maroc - Année 2015, http://www.sante.gov.ma/Documents/2016/03/Situation_\%C3\%A9pidimio_de_la_TB_au_Maroc_2015\%20Fr $\% 20 \mathrm{~V} \% 2020 \% 20 \% 20 \mathrm{mars} . \mathrm{pdf}$

7. VAUGHAN KD: Extraspinal osteoarticular tuberculosis: a forgotten entity? West Indian Med J, 2005, 54, $202-$ 206.

8. Ridley N, Shaikh Mi, Remedios D, Mitchell R. Review: radiology of skeletal tuberculosis. Orthopedics.1998;21:1213-1220.

9. Wolfgang GL: Tuberculosis Joint Infection. Clin Orthop, 1978, 136, 257-263.

10. Gillespie WJ, Mayo KM, Johnstone V: Skeletal Tubercolosis in New Zeland since the introduction of chemotherapy. Aust N Z J Surg, 1987, 57, 727-732.

11. Tuli SM. General principles of osteoarthicular tuberculosis. Clin Orthop Relat Res. 2002 May;(398):11-9.

12. Inoue S, Matsumoto S, Iwamatsu Y, Satomura M. Ankle tuberculosis: a report of four cases in a Japanese hospital. J Orthop Sci. 2004;9(4):392-8. 